\title{
Urgent Care of Fetal Heart Rate Bradycardia
}

\author{
Kazuo Maeda* \\ Professor Emeritus of Ob/Gyn, Japan
}

ISSN: 2577-2015

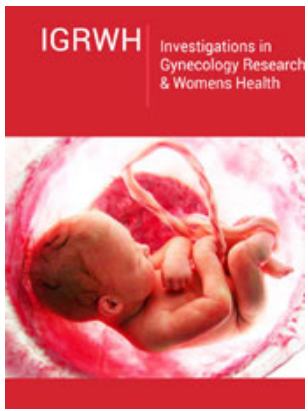

*Corresponding author: Michael Naafs, Dutch internist endocrinologist, Netherland

Submission: April 16, 2019

Published: 制 May 06, 2019

Volume 2 - Issue 5

How to cite this article: Kazuo Maeda. Urgent Care of Fetal Heart Rate Bradycardia. Invest Gynecol Res Women's Health.2(5). IGRWH.000548.2019. DOI: 10.31031/IGRWH.2019.02.000548

Copyright@ Kazuo Maeda, This article is distributed under the terms of the Creative Commons Attribution 4.0 International License, which permits unrestricted use and redistribution provided that the original author and source are credited.

\begin{abstract}
As fetal bradycardia is the sign of fetal hypoxia, emergency early caesarean section is usually indicated in fetal heartrate (FHR) in the labor, while in some cases simple lateral posture is recommended. Infantile cerebral palsy is prevented, if novel hypoxia index is 24 or less. Hypoxia index, FHR score and FHR curve frequency spectrum are update progress in automated computerized FHR diagnosis.
\end{abstract}

Keywords: FHR monitoring; Fetal bradycardia; Hypoxia index; FHR score; FHR frequency spectrum; Computer diagnosis

\section{Introduction}

As fetal heart rate decreases if fetal vagal nerve center is excited by the stimulation of low $\mathrm{PaO}_{2}$ lower than $50 \mathrm{mmHg}$ in fetal hypoxia, sudden transient or continuous fetal bradycardia, fetal hypoxic damage, particularly fetal brain damage followed by hypoxic ischemic encephalopathy (HIE) and infantile cerebral palsy in severe hypoxia, in the loss of fetal heart rate (FHR) variability, urgent care is needed in the labor. Fetal acoustic bradycardia listened after uterine contraction with stethoscope was warned to be dangerous to the beginner in old time, and also in modern fetal monitoring detected with various FHR monitor. Microbial infection with TORCH and syphilis also develop FHR change, e.g. cytomegalovirus.

\section{Result \& Discussion}

\section{Changing maternal posture to lateral one in intrapartum fetal bradycardia}

As the late deceleration (transient bradycardia) is caused by the loss of placental blood flow due to the compression of maternal iliac artery with contracted pregnant uterus (Poseiro effect), lateral posture rejects uterine compression, then the deceleration disappears [1,2]. Also, fetal deceleration appears in maternal supine hypotension caused by the compression of maternal inferior vena cava with pregnant uterus in supine posture, resulting hypotension due to the reduction of returning blood to maternal heart. Lateral posture removes vena cava compression and blood pressure returns, then fetal bradycardia disappears. Umbilical cord compression causes fetal bradycardia due to fetal hypoxia, and further continuous bradycardia appears in the cord prolapse, entanglement, or heavy torsion of umbilical cord, where the bradycardia may be improved by changing maternal posture to lateral one, however, severe cord compression may need caesarean delivery.

\section{Infantile cerebral palsy is prevented with novel hypoxia index}

The cerebral palsy is prevented, if the novel hypoxia index is 24 or less in the fetal monitoring, while cerebral palsy appears when the hypoxia index was 25 or more. The hypoxia index is the sum of deceleration durations or continuous bradycardia ( $\mathrm{min}$ ) in fetal monitoring, divided by the lowest heart rate (bpm) and multiplied by 100? The idea is based on the fact that normal neonate was born after 3 connected typical late decelerations, and by the fact of severe asphyxia and brain damage of neonate in 50 minutes' repetition of late decelerations, namely, fetal outcome was not influenced by the late appearance pattern of deceleration, but frequently repeated decelerations were effective to develop ominous outcome. The probability of wrong diagnosis was almost zero in Chi2 test of cerebral palsy case numbers in 25 or more and 24 or less hypoxia index cases, and it was significant difference, thus, it was concluded that cerebral palsy is prevented if the HI is 24 or less. Thus, 
hypoxia index should be always calculated continuously, after the first appearance of deceleration or bradycardia, to decide early cesarean delivery to prevent cerebral palsy. Fetal death is prevented also by keeping the HI below 24, because there was no fetal death in all 22 cases studied by hypoxia index in the present study, while it was also studied by the Apgar scores and UAPH predicted by FHR score [3].

\section{FHR score}

FHR score is calculated by the evaluation of FHR deceleration constructed parts in 5 minutes, according to the percentage of Apgar score lower than 7. The fetus is normal when FHR score was less than 10. The Apgar score and UAPH are predicted by their regression equations to FHR score, by FHR analysing computer. Fetal demise is predicted when estimated Apgar score is close to 0, where FHR score is 24 [3].

\section{Frequency spectrum of fetal heart rate curve}

Frequency spectrum of fetal heart rate curve is effective to automatically diagnose pathologic sinusoidal heart rate, which detects severe fetal anemia caused by geomaterial hemorrhage or ParvoB19-viral infection, which are close to fetal demise, where La/ Ta ratio was 39 or more \%, and at the same time PPSD is 300 or more $\mathrm{bpm}_{2} / \mathrm{Hz}[4]$.

\section{Conclusion}

Lateral posture should be tried in parturient woman, when a transient or continuous fetal bradycardia appears, which means the presence of fetal hypoxia. Hypoxia index should be determined continuously in the appearance of bradycardia, keeping hypoxia index at 24 or less in the delivery to prevent cerebral palsy. Apgar score \& UAPH are predicted even in the $1^{\text {st }}$ stage of labor by automated computerized fetal monitoring using FHR score preventing fetal death. Baseline frequency spectrum is analyzed to detect pathologic sinusoidal heart rate to prevent fetal demise.

\section{References}

1. Caldeyro BR, Poseiro JJ, Mendez BC, Gulin LO (1967) Effect of abnormal uterine contractions on fetal heart rate in labor, pp. 9-27.

2. Poseiro JJ, Mendez BC, Caldeyro BR (1969) Effect of uterine contractions on maternal blood flow through the placenta. Perinatal factors affecting human development. Paho Advisary Committee, pp. 161-171.

3. Maeda K, Noguchi Y, Matsumoto F, Nagasawa T (2006) Quantitative fetal heat rate evaluation without pattern classification: FHR score and artificial neural network analysis. In: Kurjak, Chervenak (Eds), Textbook of Perinatal Medicine, $2^{\text {nd }}$ edn, Informa, UK, 2: 1487-1495.

4. Maeda K, Nagasawa T (2005) Automatic computerized diagnosis of fetal sinusoidal heart rate. Fetal Diagn Ther 20(5): 328-334. 